\title{
La importancia de la resiliencia y el optimismo en los comportamientos saludables en el paciente con cáncer
}

\section{The importance of resilience and optimism in the healthy behaviors in cancer patients}

\author{
Andrea Bravo-Doddoli y Rozzana Sánchez-Aragón \\ Universidad Nacional Autónoma de México ${ }^{1}$
}

Autor para correspondencia: Rozzana Sánchez Aragón, rozzara@unam.mx.

\begin{abstract}
RESUMEN
Investigación reciente señala que los comportamientos saludables de las personas dependen de múltiples factores, entre los que destacan los psicológicos. Esto es particularmente importante en el caso de personas con cáncer, ya que de dichas conductas se deriva parte de su evolución. El presente estudio tuvo como objetivos explorar la relación entre la resiliencia y el optimismo con los comportamientos saludables, e identificar las posibles diferencias de estas variables a partir del tiempo de diagnóstico en pacientes con cáncer. Para ello, participaron 196 pacientes (varones y mujeres) cuyo tiempo de diagnóstico fue variable, quienes respondieron de manera voluntaria, confidencial y anónima la Escala de Resiliencia, la Escala de Optimismo y el Índice de Comportamientos Saludables. Los resultados demuestran que la capacidad de alcanzar metas y organizarse, la confianza en el tratamiento, el apoyo familiar, la actitud positiva, la autoeficacia, la autoconfianza y la esperanza ayudaron al paciente a desarrollar hábitos saludables y a no caer en conductas de riesgo; asimismo, se observaron diferencias a partir del tiempo de diagnóstico en la fortaleza, el apoyo social, la actitud positiva, el control interno y las conductas de cuidado debido a las diferentes emociones que se experimentan en cada etapa, como depresión, incertidumbre o estrés.
\end{abstract}

Palabras clave: Resiliencia; Optimismo; Comportamientos saludables; Tiempo de diagnóstico; Cáncer.

\begin{abstract}
Recent research indicates that people's healthy behaviors depend on multiple factors, most notably psychological ones. This is particularly important in the case of people with cancer since part of their evolution derives from these behaviors. The present study had as objectives: 1) explore the relationship between resilience and optimism with healthy behaviors and 2) identify the possible differences of these variables from the time of diagnosis in cancer patients. For this, 196 patients participated (men and women) whose diagnosis time was variable and who responded voluntarily, confidentially and anonymously to the Resilience Scale, the Optimism Scale, and the Healthy Behavior Index. The results show that the ability to achieve goals and organize, confidence in the treatment, family support, positive attitude, self-efficacy, self-confidence and hope helped the patient to develop healthy habits and not fall into risky behaviors. On the other hand, there were observed differences from time of diagnosis in strength, social support, positive attitude, internal control, and
\end{abstract}

\footnotetext{
${ }^{1}$ Facultad de Psicología, Av. Universidad 3004, Coyoacán, 04510 Ciudad de México, México.
} 
caring behaviors, due to the different emotions experienced in each stage, such as depression, uncertainty, or stress.

Key words: Resilience; Optimism; Healthy behaviors; Time of diagnosis; Cancer.

$\mathrm{E}$ 1 cáncer es un término genérico que nombra a un grupo de enfermedades que pueden afectar cualquier parte del organismo y que se caracterizan por "la multiplicación rápida de células anormales que se extienden más allá de sus límites habituales y que pueden invadir partes adyacentes del cuerpo o propagarse a otros órganos" (Organización Mundial de la Salud [OMS], 2018, p. 1). Este padecimiento es una de las principales causas de muerte en el mundo, y para el año 2030 se prevé que haya 23.6 millones de casos de cáncer (Instituto Nacional del Cáncer, 2015 [INC], 2015).

Las investigaciones actuales muestran que las alteraciones de las células son el resultado de las interacciones entre factores genéticos y tres factores externos llamados carcinógenos: 1) físicos, como por ejemplo la radiación ultravioleta y la ionizante; 2) químicos, como el humo de tabaco, los contaminantes de los alimentos (aflatoxinas) o el arsénico; 3) biológicos, como virus, bacterias y parásitos (OMS, 2018). No obstante, si se tiene un estilo de vida saludable, como comer al menos cinco tipos de verduras o frutas todos los días) (Norat, Aune, Chan y Romaguera, 2014), realizar ejercicio físico constante y no consumir alcohol y tabaco, o hacerlo moderadamente (Thomson et al., 2014), se puede reducir el riesgo de padecer cáncer y de la mortalidad en general.

Sin embargo, aún falta mucha investigación sobre los factores que contribuyen a su origen, mantenimiento y evolución, entre los cuales se hallan los psicológicos, como los relacionados con las situaciones traumáticas que se han vivido (Acinas, 2014), sin obviar que el propio diagnóstico y el tratamiento del cáncer representan paradójicamente una fuente de estrés.

Aunado a ello, los pacientes con cáncer pueden experimentar diversas emociones que variarán de acuerdo con el tiempo transcurrido desde el diagnóstico. Cuando a la persona se le diagnostica cáncer, experimentará en gran medida ansiedad y estrés (Smith et al., 2008), así como un gran temor al tratamiento y a la muerte (Cardoso, Graca, Klut, Trancas y Papoila, 2016), soledad, culpa y preocupación, lo que ocasiona que no exprese su emociones hasta que haya podido procesar tal diagnóstico, en tanto que los supervivientes -es decir, los que han sobrevivido cinco años desde el diagnóstico (INC, 2015)- suelen experimentar incertidumbre e incluso aprensión hacia la nueva normalidad que viven, aunque también pueden tener emociones más positivas, como optimismo y esperanza (Ness et al., 2013). Por consiguiente, se puede afirmar que dichas emociones se modifican en la medida en que las personas transitan por el diagnóstico, el tratamiento, la recuperación del propio tratamiento y los años posteriores; así, las respuestas emocionales de los pacientes serán importantes para un proceso exitoso (Thorne et al., 2014).

Uno de los factores psicológicos que entra en juego en el proceso de enfrentamiento de las emociones provocadas por la enfermedad es la resiliencia del paciente, a la que se considera una característica protectora de la salud (Ungar, 2008), a la que se le ha definido como una resistencia relativa a las experiencias de riesgo ambiental y la superación del estrés o la adversidad (Rutter, 2006), la cual implica una recuperación positiva ante el infortunio, el estrés o el riesgo mediante el aprendizaje que se deriva de tales experiencias (Masten, Best y Garmezy, 1990). La capacidad de recuperación puede verse como la oportunidad que tienen las personas para usar sus recursos psicológicos, sociales, culturales y físicos en el mejoramiento de su bienestar (Ungar, 2008).

Para que una persona sea resiliente en un entorno de adversidad, Saavedra (2005) propone cuatro tipos de factores que deben intervenir:

- Factores personales: rasgos de las personas, como la inteligencia verbal y matemática, la tendencia al acercamiento social y el humor positivo.

- Factores cognitivos y afectivos: características del individuo que le ayudan en el área emocional, como empatía, autoestima, mo- 
tivación al logro, sentimiento de autosuficiencia, baja desesperanza, autonomía en las acciones emprendidas y orientación a la resolución de problemas.

- Factores psicosociales familiares: ambiente familiar en el que el individuo se sienta apoyado, nutrido o socorrido, los padres estimulen y apoyen, se empleé la comunicación abierta, se tenga una estructura familiar estable, haya buenas relaciones con los pares y se cuente con el respaldo de las figuras cercanas.

- Factores socioculturales: sistema de creencias y valores y relaciones sociales, políticas y educativas en los que de manera implícita y explícita se subraye la importancia de salir adelante, de prosperar y superar la adversidad.

En el caso específico de los pacientes con cáncer, la resiliencia les ayuda a enfrentar mejor la enfermedad. Por ejemplo, en cuanto a los factores personales, el paciente resiliente tendrá un mejor ajuste psicológico (Acinas, 2014), por lo que los tratamientos tendrán menos secuelas emocionales negativas (Scrignaro, Barni y Magrin, 2011). Respecto a los factores cognitivos y afectivos, le ayudará a poseer una mayor autoestima y apreciación por la vida, así como sentimientos de calma y paz (Castilla y Vázquez, 2007), por lo que cuidarán más de sí mismos durante el tratamiento, y una vez que este se termine, tendrán un mayor compromiso con su salud (Salsman, Segerstrom, Brechting, Carlson y Andrykowski, 2009). En lo referente a los factores psicosociales familiares, se ha encontrado que cuando la familia representa una fuente de apoyo y sostén al proveer, acompañar y brindar atención y cuidado al paciente, este se sentirá mejor y cumplirá de manera más adecuada las indicaciones acerca de su tratamiento; asimismo, le estimulará a seguir adelante y a mantenerse lo más sano posible (Li, Wang, Yin, Li y Li, 2018). Respecto a los factores socioculturales, $\mathrm{Wu}, \mathrm{Liu}, \mathrm{Li}$ y Li (2016) proponen que los pacientes con un nivel educativo superior pueden acceder más fácilmente a la información sobre el cáncer a través de varios canales, como la comunicación con otros pacientes, con el personal médico y la Internet, por lo que comprenden mejor la enfermedad y obtienen así una mayor sensación de control durante el curso del tratamiento.

La razón de que la resiliencia surta efecto es porque otro factor psicológico existe: el optimismo (Wu et al., 2013), que se define como una expectativa favorable acerca de las cosas que le ocurren a una persona y lo que el futuro le promete en la vida. Es un constructo opuesto al pesimismo, y es estable temporal y situacionalmente (Scheier, Carver y Bridges, 1994), así, las personas optimistas esperan que las cosas salgan bien y, en general, creen que les sucederán cosas buenas en lugar de malas, mientras que los pesimistas esperan que las cosas salgan mal y tienden a anticipar resultados negativos (Scheier y Carver, 1985). En congruencia con lo anterior, Carver, Scheier y Segerstrom (2010) proponen que los niveles altos de optimismo se relacionan prospectivamente con un mejor bienestar subjetivo en tiempos de adversidad o dificultad, toda vez que genera el uso de conductas de afrontamiento adaptativas (Scheier y Carver, 1985) y, en consecuencia, una buena salud física (cf. Peterson, Seligman y Vaillant, 1988).

En las enfermedades como el cáncer, el optimismo desempeña un papel central al disminuir la ansiedad, el estrés (Brydon, Walker, Wawrzyniak, Chart y Steptoe, 2009; De Moor et al., 2006), la angustia (Carver et al., 2010) y la depresión (Abdel-Khalek, 2006) que puedan causar el diagnóstico y el tratamiento (Smith et al., 2008). Las personas optimistas tienen más posibilidades de aceptar de una mejor manera la realidad de la situación a la que se enfrentan, son menos propensos a hacer esfuerzos conscientes para evadir y negarse a lidiar con su enfermedad y tienden a tener expectativas positivas del tratamiento. Asimismo, el optimismo ayudará a la adopción de medidas proactivas para proteger la vida (Carver et al., 1993; Gallagher, Long, Richardson y D’Souza, 2019).

Por el lado de la salud física, los sobrevivientes de cáncer y los pacientes en tratamiento sufren más afecciones crónicas y tienen una salud más pobre (Holmes et al., 2014), por lo que las conductas que favorecen el buen estado del organismo y evitan el desarrollo de nuevas afecciones son esenciales (Kenzik, Fouad, Pisu y Martin, 2016; Shapiro, Seeff y Nadel, 2001). Asimismo, en los pacientes tiende a aumentar la probabilidad 
de fallecer no solamente por la enfermedad misma, sino también por dietas deficientes, actividad física limitada y otros comportamientos relativos al estilo de vida (Kenzik et al., 2016). De hecho, la mayoría de los pacientes no cumplen con las recomendaciones dietéticas ni con las relativas a la actividad física (Rebholz et al., 2012) y tienen un alto riesgo de que surjan otros padecimientos, por lo que es de vital importancia que obtengan información sobre cómo mantener la salud y el bienestar general (Kenzik et al., 2016), así como los elementos psicológicos suficientes para desarrollar estos comportamientos saludables (Carver et al., 2010).

Los comportamientos saludables se definen como aquellas acciones específicas para mejorar la salud, que se adquieren y aprenden a través de un proceso de identificación, atención, adquisición, práctica, mantenimiento y desarrollo. Tales conductas involucran diversas áreas de la vida de los individuos, como el trabajo, la movilidad a distintos lugares, la calidad familiar, la actividad física, la vida social, las diversas actividades caseras, los paseos, la higiene, la alimentación y la filosofía de vida, entre muchas otras (Cortés, Ramírez, Olvera y Arriaga, 2009).

Para que los comportamientos saludables se desarrollen es necesario que el paciente posea autoeficiencia (Guntzviller, King, Jensen y Davis, 2017), fortaleza (Bahrami, Mohamadirizi y Mohamadirizi, 2018), capacidad para organizarse (Wilson et al., 2004), compromiso (Putri, Wati y Rekawati, 2018) y autoconfianza (Rasmussen, Scheier y Greenhouse, 2009), mientras que en las áreas familiar y de pareja es necesario contar con un ambiente sano (Rattay et al., 2018) en el que mediante el apoyo se logren facilitar dichas conductas (Burrows, 2017). De hecho, la investigación reciente demuestra que las personas con un mayor apoyo social y familiar tienden a cuidar más las conductas relativas a su salud (Spohr, Suzuki, Marshall, Taxman y Walters, 2016).

Debido a que los comportamientos saludables resultan imprescindibles en el tratamiento del paciente con cáncer (Cormie et al., 2018; Khoshnood, Dehghan, Iranmanesh y Rayyani, 2019; Shapiro, Seeff y Nadel, 2001) y para ayudar a los sobrevivientes a no desarrollar una nueva enfermedad que pudiera complicar su condición (Kenzik et al., 2016), es evidente la importancia de las variables psicológicas para mantener estos comportamientos saludables, por lo que el presente estudio tuvo como objetivos explorar la relación entre la resiliencia y el optimismo en los comportamientos saludables de pacientes con cáncer, así como examinar las posibles diferencias en dichas variables a partir del tiempo de diagnóstico.

\section{MÉTODO}

\section{Participantes}

Se empleó una muestra no probabilística por cuota (Hernández, Fernández y Bautista, 2014) integrada por 196 participantes de la Ciudad de México, de los cuales 55 eran varones y 141 mujeres, con edades de 21 a 78 años $(M=48$, D.E. $=10.62)$. Respecto a su escolaridad, 25\% indicó contar con estudios de secundaria, 29\% de preparatoria y $46 \%$ de licenciatura. El diagnóstico de cáncer se les había dado a conocer entre un mes y 20 años atrás $(\mathrm{M}=3.85$, D.E. $=3.70)$. De ellos, $85 \%$ recibía tratamiento en ese momento. Los criterios de inclusión de los participantes fueron los de ser mayor de 18 años, tener una escolaridad mínima de secundaria y estar diagnosticado con algún tipo de cáncer al momento del estudio. Se excluyó a quienes no habían aceptado el diagnóstico, tuvieran una escolaridad menor a secundaria o no estuvieran de acuerdo con los términos del formato de consentimiento informado.

\section{Instrumentos}

\section{Escala de Resiliencia (RESI-M)}

(Palomar y Gómez, 2010).

Orientada a evaluar la resiliencia en población adulta, se utilizó la versión corta de 25 reactivos en formato de respuesta tipo Likert de cinco puntos que indican los grados de acuerdo con la afirmación, donde 1 significa "Totalmente en desacuerdo" y 5 "Totalmente de acuerdo". Este instrumento, que posee un coeficiente alfa de Cronbach total de .93, 
se compone de cinco factores obtenidos mediante el factorial de componentes principales y rotación varimax que explican $43.60 \%$ de la varianza. Dichos factores son, a saber: 1) Fortaleza y confianza en sí mismo $(\alpha=.92)$, con cinco reactivos, como "Soy una persona fuerte" o "Tengo el control de mi vida"; 2) Competencia social $(\alpha=.89)$, que consta de cinco reactivos, como "Es fácil para mí hacer reír a otras personas" o "Me es fácil establecer contacto con nuevas personas"; 3) Apoyo familiar $(\alpha=.87)$, constituido asimismo por cinco reactivos, como "Tengo una buena relación con mi familia" o "Aun en momentos difíciles nuestra familia tiene una actitud optimista hacia el futuro"; 4) Apoyo social $(\alpha=.84)$, formado por seis ítems, como "Tengo algunos amigos/familia que valoran mis habilidades" "Siempre tengo alguien que puede ayudarme cuando lo necesito", y 5) Estructura $(\alpha=.79)$, con cinco reactivos, como "Soy bueno(a) para organizar mi tiempo" y "Trabajo mejor cuando tengo metas".

Escala de Optimismo (Sánchez-Aragón, 2018).

Con un coeficiente alfa de Cronbach total de .937, este instrumento evalúa las expectativas positivas de las personas acerca de su futuro, así como una tendencia a ver la realidad por el lado más afortunado. En su versión corta consta de veinte reactivos en formato de respuesta tipo Likert de cinco puntos que indican el grado de acuerdo respecto a cada afirmación, donde 1 es "Totalmente en desacuerdo" y 5 "Totalmente de acuerdo". La validez se obtuvo a través de un análisis factorial de componentes principales y rotación ortogonal, obteniéndose cuatro factores que explicaron 55.11\% de la varianza. Estos factores son los siguientes: 1) Actitud positiva $(\alpha=.90)$, con cinco reactivos, como "Doy la bienvenida a cada nuevo reto" o "Soy optimista aunque parezca que lo que viene va a ser negativo"; 2) Control interno $(\alpha=.78)$, con cuatro ítems, como "Para cada problema encuentro una solución" y "Creo que puedo cumplir mis metas"; 3) Autoconfianza ( $\alpha=.81)$, con cinco reactivos, como "Veo el futuro con confianza" y "Tengo mucha confianza en mí mismo(a)", y 4) Esperanza $(\alpha=.80)$, con cinco reactivos, como "La mayor parte del tiempo espero que sucedan cosas buenas" o "Creo que mi futuro va a estar muy bien".

\section{Índice de Comportamientos Saludables}

(Santiago, Sánchez-Aragón y Zepeda, 2019).

Mide algunas de las conductas orientadas a mejorar y cuidar de la salud en general mediante 18 ítems, con un formato de respuesta tipo Likert de cinco puntos que indica la frecuencia con la cual se lleva a cabo cada conducta, donde 1 es "Nunca hago esto" y 5 "Siempre hago esto". Se compone de tres factores y un indicador, obtenidos a través del análisis factorial exploratorio que explica $45.46 \%$ de la varianza. Obtuvo un coeficiente alfa de Cronbach total de bajo a moderado debido a que cada factor es independiente de los otros. Dichos factores son los siguientes: 1$)$ Descuido $(\alpha=.63)$, con seis ítems, como "Cuando tengo hambre no me detengo a ver la procedencia de los alimentos" y "Uso el teléfono celular mientras manejo o camino"; 2) Prevención de adicciones $(\alpha=.69)$, con cuatro reactivos, como "Evito consumir tabaco" y "Evito consumir cualquier tipo de droga"; 3) Actividad física y relajación $(\alpha=.63)$, con seis reactivos, como "Dedico determinado tiempo a la semana para realizar actividades de ocio o recreativas" y "Hago un esfuerzo por encontrar momentos durante el día para relajarme". El indicador es Cuidado $(\alpha=.69)$, con dos ítems: "Soy cuidadoso(a) al realizar actividades que involucren cuchillos o fuego" y "Procuro caminar con precaución cuando el piso esta mojado o resbaloso".

\section{Procedimiento}

Los cuestionarios fueron aplicados por estudiantes de la carrera de Psicología entrenados, quienes acudieron a lugares de la Ciudad de México en los que se podía encontrar personas de interés (clínicas, hospitales, albergues, etc.) que cumplieran con los criterios de inclusión. Una vez que se identificó a los participantes, se les informó que la participación era voluntaria, confidencial y anónima, y asimismo se les indicó el objetivo de la investigación, advirtiéndoles que podían retirarse en cualquier momento si las preguntas afectaban su moral o creencias. Por otro lado, se les informó que sus datos serían utilizados únicamente en análisis estadísticos, por lo que no se les pediría ningún dato personal que pusiera en riesgo su identidad o privacidad, y que toda la información 
proporcionada sería tratada con las medidas de seguridad apropiadas, de conformidad con los principios contenidos en la Ley Federal de Protección de Datos Personales en Posesión de los Particulares, en su Reglamento y en los Lineamientos del Aviso de Privacidad de los Estados Unidos Mexicanos. Contando ya con su autorización, se procedió a la aplicación individual, misma que tuvo una duración de 10 minutos. Se dio respuesta a las preguntas que pudieran surgir durante la misma y se pusieron a su disposición los hallazgos de la investigación en un futuro mediato.

\section{Análisis de datos}

Se realizó la prueba de Kolmogorov-Smirnov para comprobar la normalidad de la muestra. Los resultados mostraron un rango de significancias que no cumplía los criterios de distribución para utilizar estadística paramétrica $(p \leq .024)$. Con base en ello, y para cumplir los objetivos del estudio, primeramente se obtuvo una correlación rho de Spearman para examinar si había una relación entre la resiliencia y el optimismo y los comportamientos saludables de pacientes con cáncer. Para cumplir el segundo objetivo, se efectuó un análisis Kruskal-Wallis de una vía para comprobar la diferencia entre los tres grupos con diagnóstico de cáncer, haciendo los cortes a partir de lo propuesto por Ness et al. (2013).

\section{RESULTADOS}

Para poder cumplir con el primer objetivo de esta investigación, se realizó un análisis de correlación rho de Spearman entre la resiliencia y el optimismo con los comportamientos saludables. Respecto a la variable de resiliencia, los resultados indican que en la medida en que el paciente con cáncer tenga mayor fortaleza y confianza en sí mismo (claridad sobre los objetivos, esfuerzo que hacen por alcanzar sus metas y la confianza que tienen de que van a tener éxito), generará más conductas de cuidado (p. e., de atención y preocupación por no involucrarse en acciones de riesgo), y menos de descuido (comportamientos negligentes, como no cuidar la procedencia de los alimentos e incluso favorecer aquellos con mayor riesgo para la salud). Además, a más competencia social (aptitudes para relacionarse con los demás, facilidad para hacer nuevos amigos, hacer reír a las personas y disfrutar de una conversación), más conductas de cuidado (Tabla 1).

Tabla 1. Correlaciones entre la resiliencia y el optimismo con los comportamientos saludables.

\begin{tabular}{|c|l|c|c|c|c|}
\hline \multirow{4}{*}{ Variable } & \multicolumn{2}{|c|}{ Factores } & \multicolumn{3}{c|}{ Comportamientos saludables } \\
\cline { 3 - 6 } & & Descuido & $\begin{array}{c}\text { Prevención } \\
\text { de adicciones }\end{array}$ & $\begin{array}{c}\text { Actividad física } \\
\text { y relajación }\end{array}$ & Cuidado \\
\hline \multirow{5}{*}{ Resiliencia } & Fortaleza y confianza & $-.194 * *$ & & & $.334^{* *}$ \\
\cline { 2 - 6 } & Competencia social & & & & $.280^{* *}$ \\
\cline { 2 - 6 } & Apoyo familiar & $-.265^{* *}$ & $.196 * *$ & $.271^{* *}$ & $.389^{* *}$ \\
\cline { 2 - 6 } & Apoyo social & & & & $.465^{* *}$ \\
\cline { 2 - 6 } & Estructura & & & $.232^{* *}$ & $.351^{* *}$ \\
\hline \multirow{5}{*}{ Optimismo } & Actitud positiva & & & $.299^{* *}$ & $.431^{* *}$ \\
\cline { 2 - 6 } & Control interno & & & $.290^{* *}$ & $.290^{* *}$ \\
\cline { 2 - 6 } & Autoconfianza & & & $.260^{* *}$ & $.325^{* *}$ \\
\cline { 2 - 6 } & Esperanza & & & & \\
\hline
\end{tabular}

Se observó que a más apoyo familiar (soporte, ayuda y lealtad entre los miembros de la familia y el tiempo que pasan juntos), más conductas de cuidado, actividad física y relajación (hacer ejercicio físico, beber agua, llevar a cabo actividades recreativas y de relajación que permiten a la persona mantener su cuerpo y su mente de manera integral) llevaban a cabo, así como una prevención más efectiva de adicciones (evitación del consumo de tabaco, otras drogas y alcohol) y un menor 
descuido. En la medida en que tenían un mayor apoyo social (contar con personas que puedan ayudar en momentos difíciles, que den aliento y que se preocupen por la persona), los pacientes con cáncer mostraron emprender más acciones orientadas al cuidado. Finalmente, a más estructura, esto es, capacidad para organizarse, planear las actividades y el tiempo, tener reglas y actividades sistémicas aun en momentos difíciles), más proclives se mostraron los participantes para realizar actividad física y ejercicios de relajación (ver Tabla 1).

En cuanto al optimismo, se encontró que entre mayor era su actitud positiva (predisposición esperanzadora ante los retos o situaciones negativas que se les pudiesen presentar), mayores eran su control interno (creencias de autoeficacia del individuo que indican que trabajando duro se puede lograr cualquier cosa y que cada problema tiene una solución), autoconfianza (idea sobre la certeza en las propias capacidades para resolver tareas, para influir en otros, dominar los problemas difíciles y lo que venga en el futuro), esperanza (convicción de que en el futuro la persona tendrá alimento, cuidado, hogar y personas que le brinden ayuda), cuidado y actividad física y relajación (ver Tabla 1).

Con la finalidad de cumplir el segundo objetivo de esta investigación, se efectuó una prueba de Kruskal-Wallis de una vía para identificar las posibles diferencias en la resiliencia, el optimismo y los comportamientos saludables a partir del tiempo de diagnóstico. Para crear los grupos por tiempo de diagnóstico, se hicieron algunos cortes que permitieron clasificar a los participantes del modo siguiente: 1) de un mes a 16 meses (Etapa temprana), 2) de 17 meses a 4 años, 11 meses (Etapa avanzada) y 3) de 5 años y más (Sobrevivientes) (cf. Ness et al. (2013). A partir de dicho análisis, se hallaron diferencias estadísticamente significativas que indican que los grupos de etapa avanzada y sobrevivientes mostraron tener mayor fortaleza y apoyo social (factores de resiliencia), una actitud y un control interno más positivos (factores de optimismo) y un mayor cuidado (indicador de comportamientos saludables), en comparación con el grupo en etapa temprana (Tabla 2).

Tabla 2. Prueba de Kruskal-Wallis para Resiliencia, Optimismo y Comportamientos saludables.

\begin{tabular}{|c|c|c|c|c|c|c|c|c|c|}
\hline Escala & Variable & Grupos & $\mathbf{N}$ & $\begin{array}{c}\text { Rango } \\
\text { promedio }\end{array}$ & М. & D.E. & $\chi^{2}$ & $p$ & Post-hoc \\
\hline \multirow{2}{*}{ Resiliencia } & Fortaleza & $\begin{array}{l}\text { Grupo } 1 \\
\text { Grupo } 2 \\
\text { Grupo } 3\end{array}$ & $\begin{array}{l}70 \\
59 \\
67\end{array}$ & $\begin{array}{r}80.22 \\
117.89 \\
111.13\end{array}$ & $\begin{array}{l}3.72 \\
4.26 \\
4.12\end{array}$ & .777 & 6.035 & .001 & $\begin{array}{l}\text { Grupo 1-2 } \\
\text { Grupo 1-3 }\end{array}$ \\
\hline & $\begin{array}{l}\text { Apoyo } \\
\text { social }\end{array}$ & $\begin{array}{l}\text { Grupo } 1 \\
\text { Grupo } 2 \\
\text { Grupo } 3\end{array}$ & $\begin{array}{l}70 \\
59 \\
67\end{array}$ & $\begin{array}{r}88.98 \\
113.27 \\
102.61\end{array}$ & $\begin{array}{l}4.21 \\
4.55 \\
4.37\end{array}$ & .764 & 6.054 & .024 & Grupo 1-2 \\
\hline \multirow{2}{*}{ Optimismo } & $\begin{array}{l}\text { Actitud } \\
\text { positiva }\end{array}$ & $\begin{array}{l}\text { Grupo } 1 \\
\text { Grupo } 2 \\
\text { Grupo } 3\end{array}$ & $\begin{array}{l}70 \\
59 \\
67\end{array}$ & $\begin{array}{r}88.44 \\
105.21 \\
110.53\end{array}$ & $\begin{array}{l}3.74 \\
3.95 \\
4.06\end{array}$ & .743 & 6.090 & .050 & Grupo 1-3 \\
\hline & $\begin{array}{l}\text { Control } \\
\text { interno }\end{array}$ & $\begin{array}{l}\text { Grupo } 1 \\
\text { Grupo } 2 \\
\text { Grupo } 3\end{array}$ & $\begin{array}{l}70 \\
59 \\
67\end{array}$ & $\begin{array}{r}84.44 \\
111.21 \\
111.83\end{array}$ & $\begin{array}{l}3.81 \\
4.05 \\
4.19\end{array}$ & .715 & 11.236 & .004 & Grupo 1-3 \\
\hline $\begin{array}{l}\text { Comporamientos } \\
\text { saludables }\end{array}$ & Cuidado & $\begin{array}{l}\text { Grupo } 1 \\
\text { Grupo } 2 \\
\text { Grupo } 3\end{array}$ & $\begin{array}{l}70 \\
59 \\
67\end{array}$ & $\begin{array}{r}90.19 \\
115.88 \\
98.29\end{array}$ & $\begin{array}{l}4.19 \\
4.57 \\
4.27\end{array}$ & .884 & 7.498 & .029 & Grupo 1-2 \\
\hline
\end{tabular}

Para identificar los grupos en los cuales había diferencias, se llevó a cabo una prueba de comparaciones múltiples (post-hoc), en la que se obtuvo que, en la Escala de Resiliencia, el factor de Fortaleza en los grupos de etapa avanzada y sobrevivientes difiere estadísticamente, de manera significativa, con el grupo de etapa temprana, siendo menor la media en aquella; en cuanto a los factores de Apoyo social, los grupos que difirieron estadísticamente de manera significativa fueron el de etapa temprana con etapa avanzada, teniendo una media mayor el segundo grupo en ambos factores.

En la Escala de Optimismo, en cuanto a Actitud positiva y Control interno se obtuvo una dife- 
rencia estadísticamente significativa entre el grupo de etapa temprana y el de sobrevivientes, pues en ambos casos la media en este último fue mayor. Por último, en los comportamientos saludables para el indicador de Cuidado, se obtuvo una diferencia estadísticamente significativa entre los grupos de etapa temprana y avanzada, siendo la media mayor en el segundo (ver Tabla 2).

\section{DISCUSIÓN}

El cáncer, definido por determinantes biológicos y genéticos, es una de las principales causas de muerte en el mundo (INC, 2015; OMS, 2018); sin embargo, algunas variables psicológicas desempeñan un importante papel en el origen, mantenimiento y evolución de la enfermedad (Acinas, 2014). El presente estudio se planteó como objetivos los de explorar la relación entre la resiliencia y el optimismo con los comportamientos saludables en enfermos de cáncer, y examinar las posibles diferencias en dichas variables a partir del tiempo de diagnóstico en los participantes.

En lo referente al primer objetivo, se encontró que, en la medida en que los pacientes que tienen una mayor claridad sobre sus objetivos, se esfuerzan por alcanzar sus metas y tienen confianza en sus recursos tanto para seguir el tratamiento como en los demás aspectos de su vida, tendrán asimismo un mayor cuidado de su salud y no tenderán a involucrarse en conductas de riesgo (Khoshnood et al., 2019; Shapiro et al., 2001). Lo anterior evidencia que la fortaleza y la confianza en uno mismo les proveen de creencias de autoeficacia en torno al cuidado personal de su salud, lo que favorece a su vez que muestren menos comportamientos negligentes en su alimentación, área fundamental para un paciente con cáncer (Kenzik et al., 2016).

En lo que respecta a la competencia social, se encontró que los pacientes oncológicos tenderán a prestar una mayor atención a sus conductas para no caer en situaciones de riesgo mientras mayor sea su habilidad en esta esfera de la resiliencia; es decir, cuando tienen la habilidad para relacionarse fácilmente con los demás y hacer amigos, poseerán una red social más amplia, la que a su vez es una importante fuente de apoyo. De acuerdo con Spohr et al. (2016), un soporte social de calidad disminuirá la emisión de conductas de riesgo, lo cual concuerda con los resultados obtenidos respecto al apoyo familiar y social, que muestran que cuando el paciente se encuentra positivamente vinculado con su familia, halla lealtad, armonía y ayuda mutua y mostrará menos conductas de riesgo. Así, la familia es el principal constructor de hábitos de salud (Burrows, 2017), siendo su cohesión uno de los principales mediadores para su consolidación (Rattay et al., 2018), de modo que el paciente adquirirá una mayor adherencia a una rutina de ejercicio físico (Putri et al., 2018) y mostrará menos conductas que lo pongan en riesgo (Levin, Kirby y Currie 2012). En cuanto a la prevención de adicciones, se ha visto que la convivencia familiar positiva disminuye el consumo de alcohol (Richter et al., 2009) y tabaco (Levin et al., 2012; Rattay et al., 2018). En la misma línea, el apoyo social proveniente de amigos u otras personas que rodean al paciente oncológico representan un estímulo para seguir adelante y llevar a cabo comportamientos en pro de un estado de salud positivo (Spohr et al., 2016).

Cuando el paciente tiene la capacidad para organizarse, planear sus actividades y su tiempo y se impone reglas incluso en momentos difíciles, contribuye a que realice ejercicios físicos y emprenda actividades recreativas, fundamentales para su tratamiento (Cormie et al., 2018). Esto puede entenderse mejor si se considera que las personas que son organizadas tienen la capacidad de ser más constantes en el ejercicio diario debido a que practicar tales ejercicios representa un compromiso con ellos mismos y -en este caso- con su propia salud (Wilson et al., 2004).

En cuanto al optimismo, la actitud positiva ante los retos y situaciones negativas, como el diagnóstico y los tratamientos, generará que los pacientes tengan un mayor cuidado respecto a las conductas de riesgo y emprendan una mayor actividad física, pues los pensamientos positivos y la fortaleza permiten a las personas cuidar su salud física a pesar de tales eventos negativos de la vida (Bahrami et al., 2018). En cuanto al control interno, que se refiere a la autoeficacia de los pacientes para alcanzar una meta a través del trabajo, 
este tendrá una relación positiva con el cuidado y con las actividades recreativas y el ejercicio, lo cual concuerda con lo afirmado por Guntzviller et al. (2017), quienes señalan que creer en el propio dominio se relaciona con los cuidados en la alimentación, como el comer cinco frutas y verduras diariamente, evitar los alimentos grasos y procesados, practicar ejercicios rutinariamente y mantener un peso apropiado.

De igual modo, los pacientes que tienen una mayor confianza en sí mismos tienden a realizar más conductas de cuidado de su salud; esto se debe a que la creencia de que sucederán cosas buenas debido a sus propias capacidades es un predictor para el cuidado de la salud física, incluso cuando se padece una enfermedad como el cáncer (Rasmussen et al., 2009). De la misma forma se comportó el factor de esperanza en el presente estudio, relacionándose positivamente con el aumento de cuidados ante las situaciones de riesgo, debido que dicha esperanza motiva a las personas a adquirir hábitos más saludables y un estilo de vida más activo (Scioli, Scioli-Salter, Sykes, Anderson y Fedele, 2016).

En cuanto al segundo objetivo, se encontró que, según el tiempo transcurrido desde diagnóstico, hubo diferencias en los niveles de fortaleza y apoyo social (factores de resiliencia), actitud positiva y control interno (factores de optimismo) y conductas de cuidado (indicador de conductas saludables). Para discutir los resultados es importante subrayar que cuando hay un diagnóstico de cáncer, la persona sufre ansiedad, estrés (Smith et al., 2008) y temor ante el tratamiento y la muerte, principalmente en las primeras etapas de tratamiento (Cardoso et al., 2016). Otro punto importante es que las personas experimentan una etapa difícil durante el tratamiento a consecuencia de los efectos secundarios que produce, como náusea, vómito, pérdida del apetito, pérdida del cabello, úlceras bucales, agotamiento extremo y escasa energía (American Cancer Society, 2018).

En cuanto a la resiliencia, los resultados de los análisis estadísticos muestran que cuando el tiempo de diagnóstico es menor de año y medio la persona tendrá menor fortaleza y confianza en sí misma, comparadas con las de los pacientes con más años de haberlo recibido, lo que puede ser provocado por las emociones que surgen en las personas ante la recepción de noticias que ponen en entredicho su integridad y su capacidad para resolver lo que estas implican (Gaillard, García y Aguilera, 2015), además de que pueden conjugarse con otros estados afectivos o emocionales ya existentes, como la depresión o ansiedad (Cardoso et al., 2016; Smith et al., 2008). Dichas emociones afectarán la confianza y fortaleza de los pacientes (INC, 2015). Por otro lado, quienes ya han atravesado esta etapa han aprendido de su experiencia y han demostrado ante sí mismos sus capacidades de salir adelante gracias a su entereza y ánimo, lo que los mantienen positivos a pesar de tal suceso (Ness et al., 2013). Por su parte, el apoyo social observado fue mayor en la etapa avanzada del diagnóstico que en la etapa temprana, quizá porque al inicio muchas personas no comparten lo que les sucede, y si lo hacen, es común que no sepan cómo abordarlo con sus amigos, familia y equipo de trabajo; más tarde, ya procesada la noticia, y cuando la familia percibe la necesidad de participar en los distintos aspectos del tratamiento, la percepción de contar con su apoyo se manifiesta más plenamente (INC, 2015).

En lo concerniente al optimismo, cuando ya han transcurrido más de cinco años desde el diagnóstico los pacientes muestran una actitud positiva y un control interno mayores. Los resultados indican que después de este tiempo de diagnóstico a los pacientes se les considera como sobrevivientes del cáncer, por lo cual ya han logrado desarrollar y conservar una actitud positiva de aprecio y agradecimiento por su vida, así como por estar resolviendo los problemas y situaciones que la propia enfermedad les ha presentado (Ness et al., 2013). En este mismo aspecto del control interno se vio que los sobrevivientes de cáncer mejoran sus hábitos de salud para que no se desarrolle nuevamente el propio cáncer o nuevas enfermedades (Kenzik et al., 2016), por lo que manifiestan una mayor autoeficacia que les ayudará a lograr una mejor alimentación y un ejercicio físico adecuado (Guntzviller et al., 2017).

A este respecto, las conductas de prevención de atención y preocupación por no involucrarse en acciones de riesgo resultaron menores cuando los pacientes tenían menos de año y medio de ha- 
ber sido diagnosticados, en comparación con el grupo avanzado, principalmente, tal vez debido a que pudieron haber surgido estados emocionales como depresión y ansiedad ante el diagnóstico (Cardoso et al., 2016). Ya en la etapa avanzada se ha procesado más el padecimiento y se ha aprendido a tener mayores cuidados con la salud y la enfermedad, lo que ayuda a lograr un mayor bienestar (Strine et al., 2008).

A partir de estos resultados, la presente investigación demuestra que la resiliencia y el optimismo son factores clave en la vida de los pacientes oncológicos, pues contribuyen a que desarrollen comportamientos saludables. Por su parte, la resiliencia, a través de la fortaleza y el apoyo social, provee del ánimo y del contexto social cercano para que el paciente supere su condición, en tanto que el optimismo brinda la positividad hacia el futuro $\mathrm{y}$, con ello, la recuperación de la esperanza (Matzka et al., 2016). En términos de las diferen- cias según el tiempo de diagnóstico, los datos permiten saber cuándo y cómo intervenir diferencialmente con el propósito de prestar más atención a las emociones y capacidades de los pacientes (Bahrami et al., 2018) y de sus familias, como el de afrontar el diagnóstico y el tratamiento del cáncer de maneras más positivas a través del optimismo, la resiliencia y la ejecución de comportamientos saludables.

Por lo anterior, se hace necesario seguir examinando estas variables según el tipo de cáncer de los pacientes, ya que tienen diferentes tiempos de diagnóstico, tratamiento y tasa de supervivencia. Los resultados de esta y de futuras investigaciones ayudarán al personal médico a promover la salud $y$, en última instancia, a alentar las mejores estrategias para lograr un estilo de vida saludable en los sobrevivientes (Kenzik et al., 2016) que contrarreste los efectos adversos físicos y psicológicos del cáncer y su tratamiento (Cormie et al., 2018).

\section{AGRADECIMIENTOS}

Investigación realizada gracias al Programa UNAM-PAPIIT IN304919 del proyecto "Factores protectores y de riesgo a la salud en parejas sanas y con enfermedad crónico-degenerativa".

Citación: Bravo-Doddoli, A. y Sánchez-Aragón, R. (2021). La importancia de la resiliencia y el optimismo en los comportamientos saludables en el paciente con cáncer. Psicología y Salud, Número Especial, 5-16. https://doi.org/10.25009/pys.v31i3.2724.

\section{REFERENCIAS}

Abdel-Khalek, A.M. (2006). Measuring happiness with a single-item scale. Social Behavior and Personality, 34(2), 139-150. Doi: $10.2224 /$ sbp.2006.34.2.139.

Acinas A., M.P. (2014). Situaciones traumáticas y resiliencia en personas con cáncer. Psiquiatría Biológica, 21(2), 65-71. Doi: 10.1016/j.psiq.2014.05.003.

American Cancer Society (2018). Cancer facts and figures 2018. Atlanta, GA: ACS. Recuperado de https://www.cancer.org/ research/cancer-facts-statistics/all-cancer-facts-figures/cancer-facts-figures-2018.html.

Bahrami, M., Mohamadirizi, S. y Mohamadirizi, S. (2018). Hardiness and Optimism in Women with Breast Cancer. Iranian Journal of Nursing and Midwifery Research, 23(2), 105-110. Doi: 10.4103/ijnmr.IJNMR_200_16.

Brydon, L., Walker, C., Wawrzyniak, A.J., Chart, H. y Steptoe, A. (2009). Dispositional optimism and stress-induced changes in immunity and negative mood. Brain, Behavior, and Immunity, 23(6), 810-816. Doi: 10.1016/j.bbi.2009.02.018.

Burrows, L. (2017). Children as change agents for family health. Health Education, 117(5), 498-510. Doi: 10.1108/HE-10-20160044.

Cardoso, G., Graca, J., Klut, C., Trancas, B. y Papoila, A. (2016). Depression and anxiety symptoms following cancer diagnosis: A cross-sectional study. Psychology, Health \& Medicine, 21(5), 562-570. Doi: 10.1080/13548506.2015.1125006.

Carver, C.S., Pozo, C., Harris, S.D., Noriega, V., Scheier, M.F., Robinson, D.S., Ketcham, A.S., Moffat Jr., F.L. y Clark, K.C. (1993). How coping mediates the effect of optimism on distress: A study of women with early-stage breast cancer. Journal of Personality and Social Psychology, 65(2), 375-390. Doi: 10.1037/0022-3514.65.2.375. 
Carver, C.S., Scheier, M.F. y Segerstrom, S.C. (2010). Optimism. Clinical Psychology Review, 30(7), 879-889. Doi: 10.1016/j. cpr.2010.01.006.

Castilla, C. y Vázquez, C. (2007). Emociones positivas y crecimiento postraumático en el cáncer de mama. Psicooncología, 4(2), 385-404.

Cormie, P., Atkinson, M., Bucci, L., Cust, A.E., Eakin, E., Hayes, S.C., McCarthy, A.L., Murnane, A., Patchell, S. y Adams, D. (2018). Clinical Oncology Society of Australia position statement on exercise in cancer care. Medical Journal of Australia, 209(4), 184-187.

Cortés V., E., Ramírez G., M., Olvera M., J. y Arriaga A., Y. (2009). El comportamiento de salud desde la salud: La salud como un proceso. Alternativas en Psicología, 14(20), 89-103.

De Moor, J.S., De Moor, C.A., Basen-Engquist, K., Kudelka, A., Bevers, M.W. y Cohen, L. (2006). Optimism, distress, health-related quality of life, and change in cancer antigen 125 among patients with ovarian cancer undergoing chemotherapy. Psychosomatic Medicine, 68(4), 555-562. Doi: 10.1097/01.psy.0000222379.71389.91.

Gaillard, H., García M., T. y Aguilera, A. (2015). Replication stress and cancer. Nature Reviews Cancer, 15, 276-289. Doi: $10.1038 / \mathrm{nrc} 3916$.

Gallagher, M.W., Long, L.J., Richardson, A. y D'Souza, J.M. (2019). Resilience and coping in cancer survivors: the unique effects of optimism and mastery. Cognitive Therapy and Research, 43(1), 32-44. Doi: 10.1007/s10608-018-9975-9.

Guntzviller, L.M., King, A.J., Jensen, J.D. y Davis, L.A. (2017). Self-efficacy, health literacy, and nutrition and exercise behaviors in a low-income, Hispanic population. Journal of Immigrant and Minority Health, 19(2), 489-493. Doi: 10.1007/s10903016-0384-4.

Hernández S., R., Fernández C., C. y Baptista L., P. (2014). Metodología de la investigación. México: McGraw-Hill Education.

Holmes, H.M., Nguyen, H.T., Nayak, P., Oh, J.H., Escalante, C.P. y Elting, L.S. (2014). Chronic conditions and health status in older cancer survivors. European Journal of Internal Medicine, 25(4), 374-378. Doi: 10.1016/j.ejim.2013.12.003.

Instituto Nacional del Cáncer (2015, abril 27). Estadísticas del cáncer. Bethesda, MD: INC. Recuperado de https://www.cancer. gov/espanol/cancer/naturaleza/estadisticas.

Kenzik, K.M., Fouad, M.N., Pisu, M. y Martin, M. (2016). Are long-term cancer survivors and physicians discussing health promotion and healthy behaviors? Journal of Cancer Survivorship: Research and Practice, 10(2), 271-279. Doi: 10.1007/ s11764-015-0473-8.

Khoshnood, Z., Dehghan, M., Iranmanesh, S. y Rayyani, M. (2019). Informational needs of patients with cancer: a qualitative content analysis. Asian Pacific Journal of Cancer Prevention, 20(2), 557-562. Doi: 10.31557/APJCP.2019.20.2.557.

Levin, K.A., Kirby, J. y Currie, C. (2012). Adolescent risk behaviours and mealtime routines: Does family meal frequency alter the association between family structure and risk behaviour? Health Education Research, 27(1), 24-35. Doi: 10.1093/her/ cyr084.

Li, Y., Wang, K., Yin, Y., Li, Y. y Li, S. (2018). Relationships between family resilience, breast cancer survivors' individual resilience, and caregiver burden: A cross-sectional study. International Journal of Nursing Studies, 88. Doi: 10.1016/j. ijnurstu.2018.08.011.

Masten, A.S., Best, K.M. y Garmezy, N. (1990). Resilience and development: Contributions from the study of children who overcome adversity. Development and Psychopathology, 2(4), 425-444. Doi: 10.1017/S0954579400005812.

Matzka, M., Mayer, H., Köck-Hódi, S., Moses-Passini, C., Dubey, C., Jahn, P., Schneeweiss, S. y Eicher, M. (2016). Relationship between resilience, psychological distress and physical activity in cancer patients: a cross-sectional observation study. PLOS ONE, 11(4), e0154496. Doi: 10.1371/journal.pone.0154496.

Ness, S., Kokal, J., Fee-Schroeder, K., Novotny, P., Satele, D. y Barton, D. (2013). Concerns across the survivorship trajectory: Results from a survey of cancer survivors. Oncology Nursing Forum, 40(1), 35-42. Doi: 10.1188/13.ONF.35-42.

Norat, T., Aune, D., Chan, D. y Romaguera, D. (2014). Fruits and vegetables: updating the epidemiologic evidence for the WCRF/ AICR lifestyle recommendations for cancer prevention. En V. Zappia, S. Panico, G. L. Russo, A. Budillon y F. Della Ragione (Eds.): Advances in Nutrition and Cancer (pp. 35-50). New York: Springer. Doi: 10.1007/978-3-642-38007-5_3.

Organización Mundial de la Salud (2018). Cáncer. Ginebra: OMS. Recuperado dehttps://www.who.int/es/news-room/fact-sheets/ detail/cancer.

Palomar L., J. y Gómez V., N.E. (2010). Desarrollo de una escala de medición de la resiliencia con mexicanos (RESI-M). Interdisciplinaria, 27(1), 7-22.

Peterson, C., Seligman, M.E. y Vaillant, G.E. (1988). Pessimistic explanatory style is a risk factor for physical illness: A thirty-five-year longitudinal study. Journal of Personality and Social Psychology, 55(1), 23-27. Doi: 10.1037/0022-3514.55.1.23.

Putri, N.R., Wati, D.N. y Rekawati, E. (2018). The correlation of family support and social support with the adherence to physical exercise among the older persons with hypertension. International Journal of Indonesian National Nurses Association (IJINNA), 1, 55-63. Doi: 10.32944/ijinna.v1i1.19. 
Rasmussen, H.N., Scheier, M.F. y Greenhouse, J.B. (2009). Optimism and physical health: a meta-analytic review. Annals of Behavioral Medicine, 37(3), 239-256. Doi: 10.1007/s12160-009-9111-x.

Rattay, P., Lippe, E. von der, Mauz, E., Richter, F., Hölling, H., Lange, C. y Lampert, T. (2018). Health and health risk behaviour of adolescents: Differences according to family structure. Results of the German KiGGS cohort study. PLOS ONE, 13(3), e0192968. Doi: 10.1371/journal.pone.0192968.

Rebholz, C.E., Rueegg, C.S., Michel, G., Ammann, R.A., von der Weid, N.X., Kuehni, C.E. y Spycher, B.D. (2012). Clustering of health behaviours in adult survivors of childhood cancer and the general population. British Journal of Cancer, 107(2), 234-242. Doi: 10.1038/bjc.2012.250.

Richter, M., Vereecken, C.A., Boyce, W., Maes, L., Gabhainn, S.N. y Currie, C.E. (2009). Parental occupation, family affluence and adolescent health behaviour in 28 countries. International Journal of Public Health, 54(4), 203-212. Doi: 10.1007/ s00038-009-8018-4.

Rutter, M. (2006). Implications of resilience concepts for scientific understanding. Annals of the New York Academy of Sciences, 1094, 1-12. Doi: 10.1196/annals.1376.002.

Saavedra G., E. (2005). Resiliencia: la historia de Ana y Luis. Liberabit, 11(11), 91-101.

Salsman, J.M., Segerstrom, S.C., Brechting, E.H., Carlson, C.R. y Andrykowski, M.A. (2009). Posttraumatic growth and PTSD symptomatology among colorectal cancer survivors: A 3-month longitudinal examination of cognitive processing. Psycho-Oncology, 18(1), 30-41. Doi: 10.1002/pon.1367.

Sánchez-Aragón, R. (2018). El inicio y el final de la pareja: variaciones en admiración, optimismo y pasión romántica. En R. Díaz-Loving, I. Reyes L y F. López R.: La Psicología Social en México, v. XVII (pp. 999-1016). México: Asociación Mexicana de Psicología.

Santiago S., D.Z., Sánchez-Aragón, R. y Zepeda G., G.D. (2019, 15-19 de julio). Índice de comportamientos saludables: diseño y validación. En A. Lorenzo R.: Memorias del XXXVII Congreso Interamericano de Psicología. La Habana, Cuba.

Scheier, M.F. y Carver, C.S. (1985). Optimism, coping, and health: Assessment and implications of generalized outcome expectancies. Health Psychology, 4(3), 219-247. Doi: 10.1037/0278-6133.4.3.219.

Scheier, M.F., Carver, C.S. y Bridges, M.W. (1994). Distinguishing optimism from neuroticism (and trait anxiety, self-mastery, and self-esteem): A reevaluation of the Life Orientation Test. Journal of Personality and Social Psychology, 67(6), 10631078. Doi: 10.1037/0022-3514.67.6.1063.

Scioli, A., Scioli-Salter, E.R., Sykes, K., Anderson, C. y Fedele, M. (2016). The positive contributions of hope to maintaining and restoring health: An integrative, mixed-method approach. The Journal of Positive Psychology, 11(2), 135-148. Doi: 10.1080/17439760.2015.1037858.

Scrignaro, M., Barni, S. y Magrin, M.E. (2011). The combined contribution of social support and coping strategies in predicting post-traumatic growth: A longitudinal study on cancer patients. Psycho-Oncology, 20(8), 823-831. Doi: 10.1002/pon.1782.

Shapiro, J.A., Seeff, L.C. y Nadel, M.R. (2001). Colorectal cancer-screening tests and associated health behaviors. American Journal of Preventive Medicine, 21(2), 132-137. Doi: 10.1016/S0749-3797(01)00329-4.

Smith, A.W., Reeve, B.B., Bellizzi, K.M., Harlan, L.C., Klabunde, C.N., Amsellem, M., Bierman, A.S. y Hays, R.D. (2008). Cancer, comorbidities, and health-related quality of life of older adults. Health Care Financing Review, 29(4), 41-56.

Spohr, S.A., Suzuki, S., Marshall, B., Taxman, F.S. y Walters, S.T. (2016). Social support quality and availability affects risk behaviors in offenders. Health \& Justice, 4(1), 2. Doi: 10.1186/s40352-016-0033-y.

Strine, T.W., Mokdad, A.H., Balluz, L.S., Gonzalez, O., Crider, R., Berry, J.T. y Kroenke, K. (2008). Depression and anxiety in the United States: Findings from the 2006 Behavioral Risk Factor Surveillance System. Psychiatric Services, 59(12), 13831390. Doi: 10.1176/ps.2008.59.12.1383.

Thomson, C.A., McCullough, M.L., Wertheim, B.C., Chlebowski, R.T., Martinez, M.E., Stefanick, M.L., Rohan, T.E., Manson, J.E., Tindle, H.A., Ockene, J., Vitolins, M.Z., Wactawski-Wende, J., Sarto, G.E., Lane, D.S. y Neuhouser, M.L. (2014). Nutrition and physical activity cancer prevention guidelines, cancer risk, and mortality in the Women's Health Initiative. Cancer Prevention Research, 7(1), 42-53. Doi: 10.1158/1940-6207.CAPR-13-0258.

Thorne, S., Hislop, T.G., Kim-Sing, C., Oglov, V., Oliffe, J.L. y Stajduhar, K.I. (2014). Changing communication needs and preferences across the cancer care trajectory: Insights from the patient perspective. Supportive Care in Cancer, 22(4), 10091015. Doi: 10.1007/s00520-013-2056-4.

Ungar, M. (2008). Resilience across cultures. British Journal of Social Work, 38(2), 218-235. Doi: 10.1093/bjsw/bcl343.

Wilson, P.M., Rodgers, W.M., Carpenter, P.J., Hall, C., Hardy, J. y Fraser, S.N. (2004). The relationship between commitment and exercise behavior. Psychology of Sport and Exercise, 5(4), 405-421. Doi: 10.1016/S1469-0292(03)00035-9.

Wu, G., Feder, A., Cohen, H., Kim, J.J., Calderon, S., Charney, D.S. y Mathé, A.A. (2013). Understanding resilience. Frontiers in Behavioral Neuroscience, 7, 1-10. Doi: 10.3389/fnbeh.2013.00010.

Wu, Z., Liu, Y., Li, X. y Li, X. (2016). Resilience and associated factors among mainland chinese women newly diagnosed with breast cancer. PLOS ONE, 11(12), e0167976. Doi: 10.1371/journal.pone.0167976. 